\title{
COMMENTARY
}

\section{Herbal therapy: a new pathway for the treatment of Alzheimer's disease}

\author{
Jinzhou Tian ${ }^{1,2 *}$, Jing Shi', Xuekai Zhang ${ }^{1,3}$ and Yongyan Wang ${ }^{4}$
}

\begin{abstract}
It has been a clinical challenge to treat Alzheimer's disease (AD). In the present commentary we discuss whether herbal therapy could be a novel treatment method for AD on the basis of results from clinical trials, and discuss the implications for potential therapy for AD pathophysiology. There is evidence to suggest that single herbs or herbal formulations may offer certain complementary cognitive benefits to the approved drugs. The current evidence supporting their use alone, however, is inconclusive or inadequate owing to many methodological limitations. Herbal mixtures may have advantages with multiple target regulation compared with the single-target antagonist in the view of traditional Chinese medicine. Several clinical trials using herbal mixtures are being conducted in China and will hopefully show promising results for treating $A D$ in the near future.
\end{abstract}

\section{Introduction}

The ultimate aim of Alzheimer's disease (AD) therapy is to stop or slow down the disease progression. Cholinesterase inhibitors have a modest clinical effect on the symptoms, however, and memantine - the currently available $N$-methyl-D-aspartate receptor antagonist does not prevent the deterioration of dementia $[1,2]$. Finding an effective method to treat $\mathrm{AD}$ still poses a significant clinical challenge.

Herbal medicine has long been used in China as therapy for dementia. The Complete Work of Jingyue published in 1624 contains the earliest known description in the world of a herbal therapeutic strategy for dementia. In the past 10 years, however, herbal drugs have seldom been approved for use alone in treating dementia.

${ }^{*}$ Correspondence: jztian@hotmail.com

'BUCM Neurology Centre, Dongzhimen Hospital, Beijing University of Chinese Medicine, Beijing 100700, China

Full list of author information is available at the end of the article
Overall, systematic review has identified a few single herbs and herbal formulations as possible effective medicine for $\mathrm{AD}$ (Table 1). According to the current evidence, some of these therapies show promising results in terms of their cognitive benefits. In the present commentary we discuss whether herbal therapy could be a novel pathway to treat $\mathrm{AD}$, on the basis of the results from clinical trials, and the implications for potential therapy of $\mathrm{AD}$ pathophysiology.

\section{Single herbs or extracts from herbs Ginkgo biloba}

Ginkgo biloba extract is among the most widely used complementary therapies. A Cochrane review included 36 trials of gingko biloba, but most trials were small and of duration $<3$ months [3]. Nine trials were of 6 months duration and of adequate size, and were conducted to a reasonable standard. Of the four most recent trials to report results, three studies found no difference between Ginkgo biloba, at different doses, and placebo [3], and one study found very large treatment effects in favor of Ginkgo biloba, but the trial sample size was very small [4]. Another recent trial reported negative results in reducing cognitive decline in older adults with normal cognition or with mild cognitive impairment [5]. The current overall evidence that Ginkgo has a predictable and clinically significant benefit for people with dementia or cognitive impairment therefore seems inconsistent and unreliable.

\section{Serrate clubmoss}

Huperzine A extracted from the serrate clubmoss herb is a potent, reversible and selective inhibitor of acetylcholinesterase. Considering the available evidence from six trials, Huperzine A seems to have some beneficial effects on improvement of general cognitive function, global clinical status, behavioral disturbance and functional performance, with no obvious serious adverse events for patients with $\mathrm{AD}$ [6]. Only one study was of adequate quality and size, but the period during this study that found very large treatment effects was only 12 weeks [7]. Overall the current evidence supporting clinical use of Huperzine A is presently inconclusive or inadequate. 
Table 1. Different herbs and formulations effective in the treatment of Alzheimer's disease

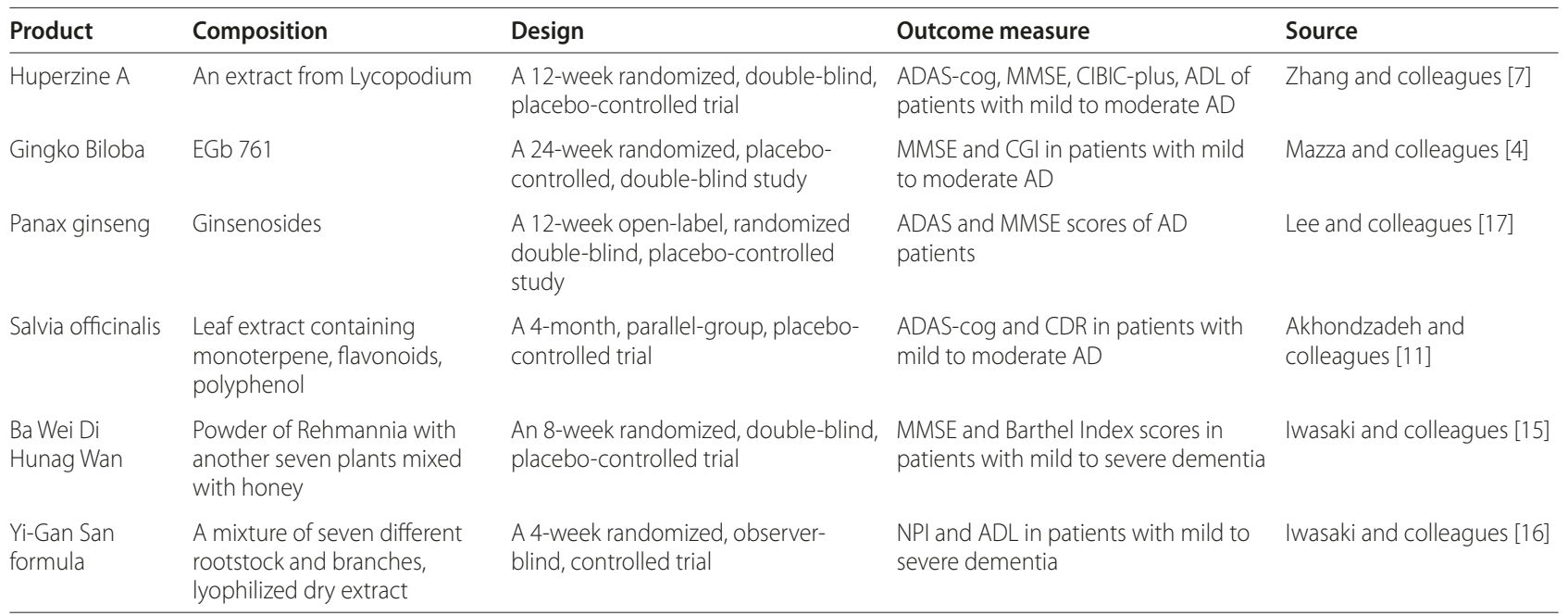

AD, Alzheimer disease; ADAS-cog, Alzheimer disease assessment scale-cognitive subscale; ADL, activities of daily living; CDR, Clinical Dementia Rating; CGI, Clinical Global Impression; CIBIC-plus, Clinician's Interview-Based Impression of Change plus Caregiver Input; MMSE, mini-mental state examination; NPI, neuropsychiatric Inventory.

\section{Ginseng}

Panaxi ginseng's main active ingredient is panaxsaponin, which can enhance psychomotor and cognitive performance, and can benefit AD by improving brain cholinergic function, reducing the level of $A \beta$ and repairing damaged neuronal networks [8]. The high-dose ginseng group showed statistically significant improvement on the Alzheimer Disease Assessment Scale (ADAS) and Clinical Dementia Rating (but not on the Mini-Mental State Examination) at the end of the study, when compared with the control group. This study was poorly designed, with an insufficient description of randomization and without blinding. Furthermore, the sample size was small ( $n=15$ for each group), and there was also a confounding effect due to concurrently administered western medications [9]. The evidence for ginseng as a treatment of $\mathrm{AD}$ is thus scarce and inconclusive. Further rigorous trials seem warranted [10].

\section{Salvia officinalis}

Salvia officinalis has been used in herbal medicine for many centuries. After 4 months of treatment, salvia officinalis extract produced a significantly better outcome on cognitive functions than placebo - as seen on the ADAS cognitive subscale and the Clinical Dementia Rating Sum of Boxes scale in patients with mild to moderate AD aged between 65 and 80 years [11]. There were no significant differences between salvia officinalis and placebo in terms of the observed side effects. In addition, salvia officinalis may reduce agitation in patients. More high-quality large-scale randomized controlled trials are needed, however, for further determination of the herb's efficacy [11].

\section{Herbal formulations or mixtures of herbal ingredients}

Herbal formulations or mixtures of herbal ingredients may have advantages with multiple target regulation compared with the single target antagonist in the view of traditional Chinese medicine, although there have been few clinical trials examining the efficacy and safety of herbal formulations in $\mathrm{AD}$ patients.

Shenwu capsule, a mixture of six herbs that is thought to reduce amyloid cytotoxicity, increased the memory score from baseline $(n=83)$ - but without significant difference from aniracetam $(n=83)$ - in a 12-week phase II trial for patients with mild cognitive impairment [12]. A phase III trial is now underway. Stilbene glycoside, an extract of Shenwu capsule, has been evaluated in a phase I trial for AD. Further results for both of these formulations will be available in the next few years.

GEPT, a combination of five active components extracted from Chinese herbs, may be valuable for the treatment of $A D$ - reducing the level of $A \beta$ via the inhibition of $\gamma$ secretase (presenilin-1) and the promotion of insulindegrading enzyme and neprilysin, which has been reported in the brain of APPV717I transgenic mice [13]. A 24-week preliminary study of GEPT showed a significant improvement on cognitive function in patients with amnestic mild cognitive impairment, an early stage of $\mathrm{AD}(n=101)$, consistently across different cognitive scales; for example, an improvement in the ADAS cognitive subscale from baseline of -4.19 points (95\% confidence interval $=-5.74$ to -2.63$)$, which declined at 24 weeks of follow-up after the GEPT withdrawal. This level of efficacy was comparable with that of -4.23 points found in the subjects taking Donepezil $(n=100)$ 


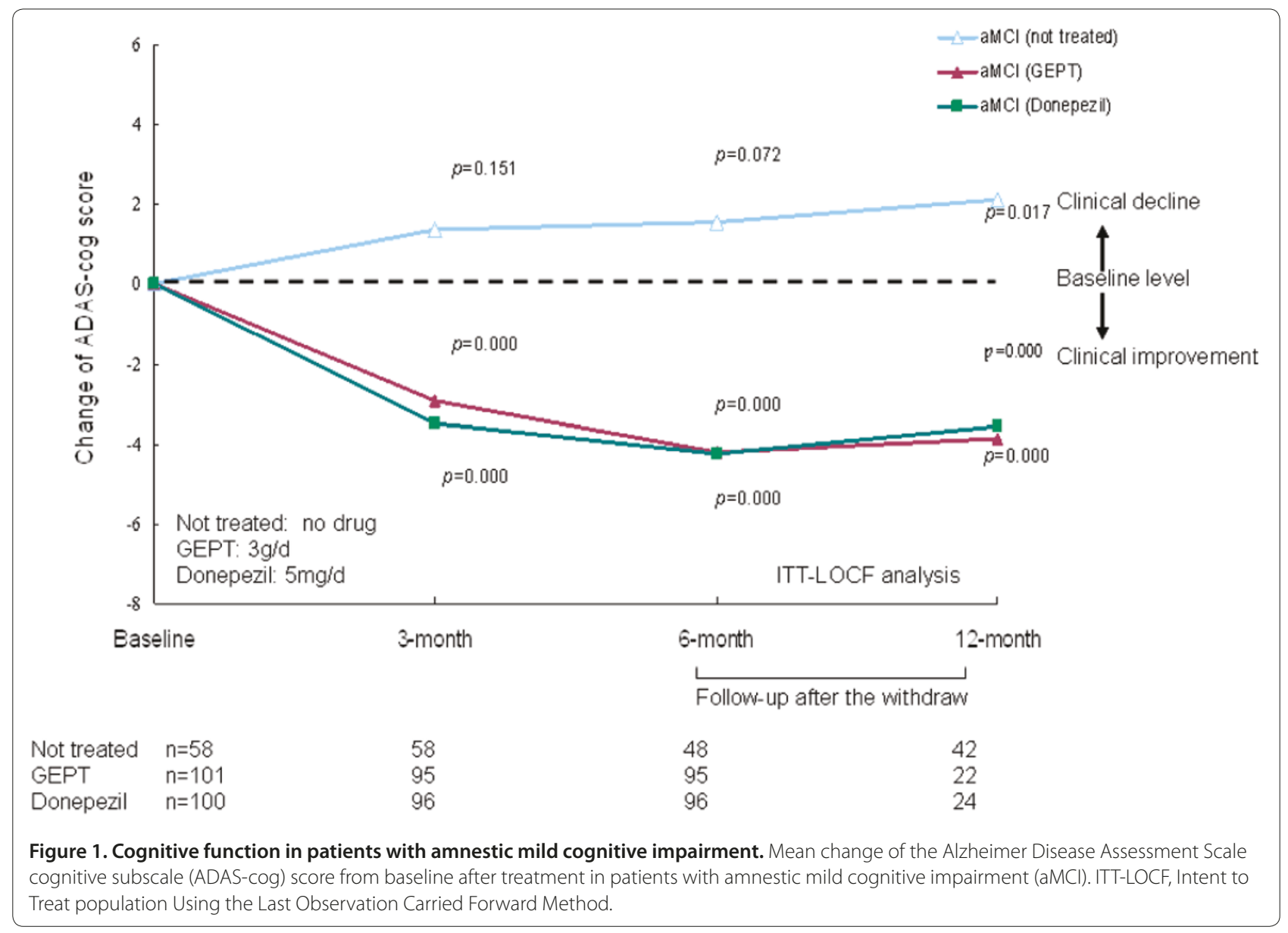

(Figure 1) [14]. GEPT is planned to apply for a shape II trial.

Furthermore, the herbal preparations Ba Wei Di Huang Wan and Yi-Gan San are individually reported to significantly improve cognition or behavior and function on the Mini-Mental State Examination, the Neuropsychiatric Inventory and the Barthel Index in the patients with $\mathrm{AD}$ $[15,16]$.

\section{Conclusion}

Single herbs or formulations may be able to complement approved drugs for $\mathrm{AD}$. No serious adverse events have been reported. The current evidence to support their use alone, however, is inconclusive or inadequate. This uncertainty is mainly caused by methodological limitations such as poor study design, relatively small sample sizes without a power calculation, inappropriate outcome measures and primary and secondary end-point selection, and invalid statistical analysis. In addition, the herbs' potential value for prevention and treatment of $\mathrm{AD}$ only results from symptomatic changes and short treatment periods ( $<6$ months). Several studies currently underway or in early-stage development in China to evaluate herb mixtures will hopefully show promising results in the near future.

\section{Abbreviations \\ AD, Alzheimer disease; ADAS, Alzheimer Disease Assessment Scale.}

\section{Competing interests}

The authors declare that they have no competing interests.

\section{Acknowledgements}

The present work was supported by the 111 Project (No. B08006), the Beijing Cooperative Study Project of the Capital Foundation of Medical Developments (No. 2005-SF-1-007), New Century Excellent Talents in University (No.

NCET-07-0117) and the Innovative Research Team of Alzheimer's Disease and Other Neurodegenerative Diseases of the Ministry of Education of China (No. IRT-08-010).

\section{Author details}

'BUCM Neurology Centre, Dongzhimen Hospital, Beijing University of Chinese Medicine, Beijing 100700, China. ${ }^{2}$ Department of Preclinical Medicine, Hubei University of Chinese Medicine, Wuhan 430065, China. ${ }^{3} \mathrm{Clinical}$ Neuroscience Research Group, The University of Manchester, Hope Hospital, Stott Lane, Salford, Manchester M6 8HD, UK. ${ }^{4}$ Institute of Clinical Medicine, China

Academy of Chinese Medical Sciences, Beijing 100700, China.

Published: 22 October 2010

\section{References}

1. Reisberg B, Doody R, Stöffler A, Schmitt F, Ferris S, Möbius HJ; Memantine Study Group: Memantine in moderate-to-severe Alzheimer's disease. 
N Engl J Med 2003, 348:1333-1341.

2. Porsteinsson AP, Grossberg GT, Mintzer J, Olin JT; Memantine MEM-MD-12 Study Group: Memantine treatment in patients with mild to moderate Alzheimer's disease already receiving a cholinesterase inhibitor: a randomized, double-blind, placebo-controlled trial. Curr Alzheimer Res 2008, 5:83-89.

3. Birks J, Grimley Evans J: Ginkgo biloba for cognitive impairment and dementia. Cochrane Database Syst Rev 2009, 1:CD003120.

4. Mazza M, Capuano A, Bria P, Mazza S: Ginkgo biloba and donepezil: a comparison in the treatment of Alzheimer's dementia in a randomized placebo-controlled double-blind study. Eur J Neurol 2006, 13:981-985.

5. Snitz BE, O'Meara ES, Carlson MC, Arnold AM, Ives DG, Rapp SR, Saxton J, Lopez OL, Dunn LO, Sink KM, DeKosky ST; Ginkgo Evaluation of Memory Study I: Ginkgo biloba for preventing cognitive decline in older adults: a randomized trial. JAMA 2009, 302:2663-2670.

6. Li J, Wu HM, Zhou RL, Liu GJ, Dong BR. Huperzine A for Alzheimer's disease. Cochrane Database Syst Rev 2008, 2:CD005592.

7. Zhang Z, Wang X, Chen Q, Shu L, Wang J, Shan G: Clinical efficacy and safety of huperzine Alpha in treatment of mild to moderate Alzheimer disease, a placebo-controlled, double-blind, randomized trial [in Chinese]. Zhonghua Yi Xue Za Zhi 2002, 82:941-944.

8. Chen F, Eckman FA, Eckman CB: Reductions in levels of the Alzheimer's amyloid beta peptide after oral administration of ginsenosides. Faseb $\mathrm{J}$ 2006, 20:1269-1271

9. Fu LM, Li JT: A systematic review of single Chinese herbs for Alzheimer's disease treatment. Evid Based Complement Alternat Med 2009, eCAM 2009: Page 1 of 8. doi:10.1093/ecam/nep136

10. Lee MS, Yang EJ, Kim Jl, Ernst E: Ginseng for cognitive function in Alzheimer's disease: a systematic review. J Alzheimers Dis 2009, 18:339-344.

11. Akhondzadeh S, Noroozian M, Mohammadi M, Ohadinia S, Jamshidi AH,
Khani M: Salvia officinalis extract in the treatment of patients with mild to moderate Alzheimer's disease: a double blind, randomized and placebocontrolled trial. J Clin Pharm Ther 2003, 28:53-59.

12. Zhong J, Tian JZ, Zhu AH, Yang CZ: Clinical study on a randomized, doubleblind control of Shenwu gelatin capsule in treatment of mild cognitive impairment [in Chinese]. Zhongguo Zhong Yao Za Zhi 2007, 32:1800-1803.

13. Tian J, Shi J, Zhang L, Yin J, Hu Q, Xu Y, Sheng S, Wang P, Ren Y, Wang R, Wang $Y:$ GEPT extract reduces a beta deposition by regulating the balance between production and degradation of amyloid-beta in APPV7171 transgenic mice. Curr Alzheimer Res 2009, 6:1 18-131.

14. Miao YC. Part 5: a randomized, double-blind and parallel control study of GEPT extract in the treatment of amnestic mild cognitive impairment. PhD thesis. Beiijing University of Chinese Medicine; 2008:95-117.

15. Iwasaki K, Kobayashi S, Chimura Y, Taguchi M, Inoue K, Cho S, Akiba T, Arai H, Cyong JC, Sasaki H: A randomized, double-blind, placebo-controlled clinical trial of the Chinese herbal medicine 'Ba wei di huang wan' in the treatment of dementia. J Am Geriatr Soc 2004, 52:1518-1521.

16. Iwasaki K, Satoh-Nakagawa T, Maruyama M, Monma Y, Nemoto M, Tomita N, Tanji H, Fujiwara H, Seki T, Fujii M, Arai H, Sasaki H: A randomized, observerblind, controlled trial of the traditional Chinese medicine yi-gan san for improvement of behavioral and psychological symptoms and activities of daily living in dementia patients. J Clin Psychiatry 2005, 66:248-252.

17 Lee ST, Chu K, Sim JY, Heo JH, Kim M: Panax ginseng enhances cognitive performance in Alzheimer disease.. Alzheimer Dis Assoc Disord 2008, 22:222-226.

doi:10.1186/alzrt54

Cite this article as: Tian J, et al.: Herbal therapy: a new pathway for the treatment of Alzheimer's disease. Alzheimer's Research \& Therapy 2010, 2:30. 